$>$ De nombreuses maladies génétiques ou neurodégénératives sont consécutives à un repliement défectueux de protéines entraînant leur dégradation ou leur accumulation sous forme d'agrégats. Ces maladies sont regroupées sous le terme de «pathologies conformationnelles ». Les protéines impliquées n'étant pas correctement repliées, elles sont disqualifiées par le système contrôle-qualité cellulaire. En conséquence, elles ne peuvent plus exercer leur rôle physiologique. Des composés spécifiques (ligands, substrats, inhibiteurs), appelés chaperons pharmacologiques, sont capables de se lier à ces protéines défectueuses, de les stabiliser afin de leur permettre d'acquérir une conformation quasi native, d'échapper au système contrôle-qualité et par conséquent de récupérer une fonctionnalité. Ces molécules ont une activité intrinsèque variable: elles peuvent être agonistes (activatrices), antagonistes (inhibitrices) ou modulateurs allostériques de leurs récepteurs, canaux ioniques ou enzymes cibles. Les chaperons pharmacologiques représentent donc un espoir thérapeutique pour des maladies rares comme la mucoviscidose, certaines rétinites pigmentaires, le diabète insipide néphrogénique, la maladie de Fabry ou de Gaucher, mais aussi pour certains cancers et des pathologies du système nerveux très invalidantes comme les maladies d'Alzheimer ou de Parkinson. <

\section{Chaperons pharmacologiques}

\section{Un espoir thérapeutique pour les pathologies conformationnelles}

Christiane Mendre, Bernard Mouillac

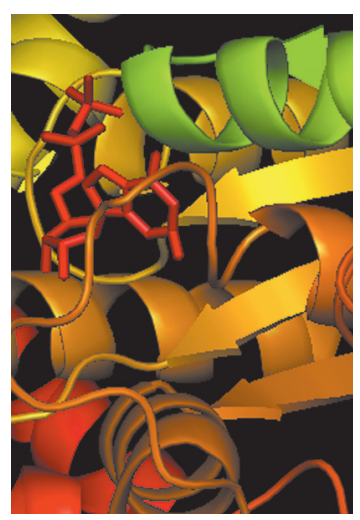

Institut de génomique fonctionnelle, CNRS UMR 5203, Inserm U661, Universités Montpellier 1 et 2, 141, rue de la Cardonille, 34094 Montpellier Cedex 05, France. christiane.mendre@igf.cnrs.fr bernard.mouillac@igf.cnrs.fr vironnement cellulaire local, est cruciale. Elle peut s'effectuer de façon coordonnée à la synthèse de la protéine considérée. Le repliement des protéines prend place dans trois compartiments cellulaires: le cytoplasme, le réticulum endoplasmique (RE) et les mitochondries. Nous nous limiterons dans cette revue au processus de repliement qui intervient dans le RE, point de départ de la voie de sécrétion des protéines vers le milieu extracellulaire, de l'export des protéines vers la membrane plasmique ou du ciblage vers d'autres compartiments membranaires impliqués dans la sécrétion ou l'endocytose [1,2]. Le RE est le siège d'un système contrôle-qualité des protéines qui fait intervenir de nombreux chaperons moléculaires et enzymes. Le rôle de ceux-ci est de reconnaître les protéines incomplètement ou mal repliées, de catalyser leur repliement efficace lors de plusieurs étapes - par exemple l'établissement de ponts disulfures ou l'accrochage de chaînes glycosylées - et de les assembler si nécessaire $[1,2]$. Ces chaperons moléculaires sont endogènes à la cellule [3], par opposition aux chaperons chimiques ou pharmacologiques que nous définirons plus loin. Une fois synthétisées et correctement repliées, les protéines rejoignent le compartiment cellulaire dans lequel elles jouent leur rôle physiologique, qu'il s'agisse de la membrane pour des récepteurs ou des canaux ioniques, du lysosome 


\section{Principales caractéristiques de pathologies conformationnelles prototypes}

Pour une recherche plus générale sur les maladies rares, se connecter à http://www.orphanet.org

Mucoviscidose $[19,20]$

- Transmission autosomique récessive.

- Signes cliniques: affection des épithéliums glandulaires de nombreux organes, augmentation de la viscosité du mucus et de son accumulation dans les voies respiratoires et digestives.

- Altération (mutations ou délétions du gène) de la protéine CFTR (cystic fibrosis transmembrane conductance regulator) qui constitue un canal ionique perméable au chlore. Canal muté non exporté à la surface cellulaire et finalement dégradé par le protéasome (perte de fonction).

Maladie de Fabry [21, 22]

- Transmission récessive liée au chromosome X.

- Signes cliniques: douleurs des extrémités, signes dermatologiques (angiokératomes), puis maladie de surcharge multiviscérale (hypertrophie ventriculaire gauche, accidents vasculaires cérébraux, hypoacousie, insuffisance rénale).

- Déficit en $\alpha$-galactosidase A (mutations ponctuelles ou délétions du gène), enzyme impliqué dans le métabolisme des glycosphingolipides. Accumulation du substrat non dégradé dans les tissus et le plasma. Dégradation précoce de l'enzyme qui n'est pas ciblée vers le compartiment lysosomial (perte de fonction).

\section{Maladie de Gaucher [23, 24]}

- Transmission autosomique récessive.

- Signes cliniques : surcharge lysosomiale due à des dépôts de glucosylcéramide (ou glucocérébroside) dans les cellules du système réticulo-endothélial du foie, de la rate et de la moelle osseuse. La manifestation la plus classique, le type 1 , chronique, non neurologique, concerne $95 \%$ des cas: organomégalie (rate, foie), atteinte osseuse (douleurs, infarctus osseux, ostéonécrose) et cytopénies (thrombopénie, anémie et plus rarement neutropénie).

- Déficit en glucocérébrosidase (appelée aussi glucosylcéramidase ou $\beta$-glucosidase acide) suite à des mutations ou délétions du gène correspondant. Dégradation précoce de l'enzyme qui n'est pas ciblée vers le compartiment lysosomial (perte de fonction).
Rétinite pigmentaire (RP) [25]

- Transmission autosomique dominante, $15 \%$ des dégénérescences rétiniennes génétiquement transmissibles.

- Signes cliniques : perte de vision périphérique et de vision de nuit pour ensuite évoluer vers une cécité totale.

- Altérations (mutations ou délétions du gène) de la rhodopsine, récepteur membranaire situé au niveau des cellules photoréceptrices de la rétine. Dégradation précoce et/ou phénomène de saturation de la maturation, du transport et de la dégradation de la rhodopsine qui s'accumule dans la cellule (exemple de synergie entre perte et gain de fonction).

Diabète insipide néphrogénique congénital (DINc) [26]

- Transmission récessive liée au chromosome X.

- Signes cliniques: polyurie (incapacité à concentrer les urines), polydipsie, hypernatrémie. Épisodes répétés de déshydratation, retard de croissance et retard mental chez les jeunes enfants. Évolution vers une insuffisance rénale si les épisodes de déshydratation sont trop fréquents ou suite à une néphropathie de reflux due au débit urinaire considérable.

- Altérations (mutations ou délétions du gène) du récepteur V2 (V2R) de la vasopressine situé au niveau de la membrane basale des cellules principales du canal collecteur du rein. Séquestration du V2R dans le réticulum endoplasmique; il n'est donc pas ciblé à la membrane plasmique (perte de fonction).

Hypogonadisme hypogonadotrope (HH) [15]

- Transmission autosomique récessive.

- Signes cliniques: impossibilité des cellules gonadotropes de I'hypophyse antérieure de répondre à l'hormone GnRH (gonadotropin-releasing hormone) libérée par l'hypothalamus. Peu ou pas de libération des hormones gonadotrophines LH (luteinizing hormone) et FSH (follicle stimulating hormone), perte de la régulation de la stéroïdogenése au niveau des organes génitaux, défaillance du système de reproduction.

- Altérations (mutations ou délétions du gène) du récepteur de la GnRH situé à la membrane plasmique des cellules gonadotropes de l'hypophyse. Rétention du récepteur GnRH dans le réticulum endoplasmique (perte de fonction). pour de nombreuses enzymes du métabolisme, ou du milieu extracellulaire pour des protéines sécrétées (anticorps par exemple). Si une protéine n'acquiert pas sa structure native malgré l'action du système contrôle-qualité, elle est dirigée vers le cytoplasme à travers le translocon et dégradée par le protéasome [4]. Dans ce cas, la protéine ne pouvant pas exercer son rôle physiologique, on parle de «perte de fonction». D'autres molécules mal repliées s'accumulent dans les cellules ou dans les compartiments extracellulaires, et y forment des agrégats : c'est le cas du peptide $\beta$-amyloïde responsable de la maladie d'Alzheimer. On parle alors de «gain de fonction», ce qui peut paraître paradoxal puisqu'en général cette accumulation de protéines inactives a des conséquences cellulaires toxiques. Dans certains cas, une perte de fonction et un gain de fonction peuvent agir de concert pour provoquer un état pathologique (la rétinite pigmentaire par exemple, voir plus loin).

Le repliement défectueux de protéines qui caractérise les maladies dites «conformationnelles» $[5,6]$ dont il sera question dans cette revue résulte de la présence de mutations dans les gènes correspondants de ces protéines. Ces mutations sont transmissibles de génération en génération ou acquises spontanément. Quelques exemples typiques de ces maladies sont décrits dans l'Encadré I et le Tableau I en donne une liste plus exhaustive. Elles mettent en cause des protéines majeures de la signalisation ou du métabolisme, comme des canaux ioniques, des enzymes et des récepteurs 


\begin{tabular}{|c|c|c|c|}
\hline Maladie/anomalie & Protéine déficiente & Mutation* & Référence \\
\hline \multicolumn{4}{|l|}{ Repliement inefficace et dégradation } \\
\hline Mucoviscidose & Canal CFTR & $>1500, \Delta \mathrm{F} 508$ & {$[8]$} \\
\hline Gaucher & $\beta$-glucosidase acide & $>200, N 370 S$ & {$[11,12]$} \\
\hline Fabry & $\alpha$-galactosidase A & $>$ 200, R301Q, Q279ع & {$[9,10]$} \\
\hline Déficience en $\alpha 1$-antitrypsine & $\alpha 1$-antitrypsine & $>90, \varepsilon 342 \mathrm{~K}, \varepsilon 264 \mathrm{~V}$ & [27] \\
\hline \multirow[t]{2}{*}{ Cancer } & $\mathrm{p} 53$ & $>1500$, V143A, G245S, R249S & {$[28,29]$} \\
\hline & Smo & $>20, W 535 \mathrm{~L}$ & {$[30]$} \\
\hline Hyperinsulinémie & Récepteur sulfonylurée SURI & $>150, \mathrm{R} 1394 \mathrm{H}, \mathrm{R} 74 \mathrm{~W}, \varepsilon 128 \mathrm{~K}$ & {$[31,32]$} \\
\hline Menkes $\S$ & ATPase MNK & $>12, \mathrm{G} 1019 \mathrm{D}$ & [33] \\
\hline Diabète insipide néphrogénique & Récepteur V2 de la vasopressine & $>200$, L44P, Y128S, A294P, R337X & {$[20,22]$} \\
\hline Diabète insipide néphrogénique & Aquaporine-2 & $>30, \mathrm{~T} 126 \mathrm{M}, \mathrm{Al} 47 \mathrm{~T}, \mathrm{R} 187 \mathrm{C}$ & [15] \\
\hline Hypogonadisme hypogonadotrope & Récepteur du GnRH & $>20, \mathrm{R} 139 \mathrm{H}, \mathrm{C} 200 \mathrm{y}, \mathrm{Y} 284 \mathrm{C}$ & [16] \\
\hline Rétinite pigmentaire & Rhodopsine & $>150, \mathrm{G} 188 \mathrm{R}$ & [34] \\
\hline Hirschsprung** & Récepteur de l'endothéline ETB & $>20$, C109R, W276C, S390R, P383L & [35] \\
\hline Obésité & Récepteur de la mélanocortine MC4 & $>80, \mathrm{C} 84 \mathrm{R}, \mathrm{W} 174 \mathrm{C}$ & {$[36]$} \\
\hline Déficit en glucocorticoïdes & Récepteur de la corticotropine MC2 ou ACTHR & $>35, \mathrm{Gl} 166 \mathrm{~V}, \mathrm{R} 137 \mathrm{~W}, \mathrm{R} 146 \mathrm{H}, \mathrm{Y} 254 \mathrm{C}$ & [37] \\
\hline Résistance à l'infection VIH & Récepteur des chimiokines CCR5 & $>20$, CCR5 $\Delta 32, \mathrm{Cl} 178 \mathrm{R}$ & [38] \\
\hline $\begin{array}{l}\text { Hypercalcémie, hypocalciurie, } \\
\text { Hyperparathyroïdisme }\end{array}$ & Récepteur sensible au calcium (CaSR) & >60, L13P, ع297K & [39] \\
\hline Hypothyroïdisme congénital & $\begin{array}{l}\text { Récepteur de l'hormone de stimulation } \\
\text { de la thyroïde (TSHR) }\end{array}$ & $>30$, I167N, L252P, T477I & {$[40]$} \\
\hline $\begin{array}{l}\text { Pseudohermaphrodisme masculin - } \\
\text { Hypogonadisme hypergonadotrope }\end{array}$ & $\begin{array}{l}\text { Récepteur de l'hormone } \\
\text { lutéinisante (LHR) }\end{array}$ & $>25, \mathrm{~V} 144 \mathrm{~F}, \mathrm{~A} 593 \mathrm{P}$ & {$[41]$} \\
\hline $\begin{array}{l}\text { Arrêt de la maturation des follicules } \\
\text { ovariens, arrêt de la spermatogenèse }\end{array}$ & Récepteur de l'hormone folliculaire (FSHR) & $>10, \mathrm{~A} 189 \mathrm{~V}, \mathrm{P} 519 \mathrm{~T}$ & [42] \\
\hline Anomalies de la peau et des cheveux & Récepteur de la mélanocortine $\mathrm{MCl}$ & $>60, \mathrm{R} 151 \mathrm{C}, \mathrm{R} 160 \mathrm{~W}$ & [43] \\
\hline \multicolumn{4}{|l|}{ Repliement inefficace et agrégation } \\
\hline Rétinite pigmentaire & Rhodopsine & $>150, \mathrm{P} 23 \mathrm{H}$ & {$[44]$} \\
\hline Parkinson & $\alpha$-synucléine & 3, A30P, A53T, ع46K & {$[45]$} \\
\hline Alzheimer & $\begin{array}{l}\beta \text {-amylö̈de, préséniline } 1 \text { et } 2 \\
\alpha 2 \text {-macroglobuline }\end{array}$ & mutations ponctuelles et délétions & {$[46]$} \\
\hline
\end{tabular}

Tableau I. Quelques exemples de pathologies conformationnelles, protéines et mutations impliquées. * La colonne de ce tableau indique le nombre de

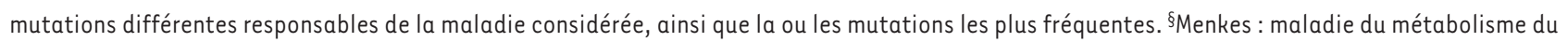
cuivre. Elle se caractérise cliniquement par un retard de croissance anté et post-natal, une détérioration neurologique progressive qui débute dans les deux premiers mois de vie, une hypotonie axiale, une spasticité, une hypothermie, des difficultés d'alimentation, des convulsions partielles ou généralisées (source : Orphanet). **Hirschsprung : malformations du tube digestif ( $1 / 5000$ naissances) résultant d'une anomalie du développement du système nerveux entérique. Elle se définit par l'absence de cellules ganglionnaires assurant l'innervation intrinsèque des couches musculaires de l'intestin terminal. Elle se traduit par une occlusion intestinale basse ou une constipation opiniâtre (source: Orphanet). 
membranaires de la famille des récepteurs couplés aux protéines G (RCPG) [7]. Ces différentes catégories de protéines sont soumises de façon très stricte au système contrôle-qualité du RE avant qu'elles ne s'engagent dans la voie de sécrétion et rejoignent leur compartiment cellulaire de fonction.

\section{Chaperons chimiques et pharmacologiques}

Extrêmement efficace dans la plupart des cas, le système contrôle-qualité du RE peut se révéler «trop» parfait. Des mutations qualifiées de mineures, ne compromettant pas la fonctionnalité des protéines impliquées, provoquent cependant soit leur déroutage et leur dégradation provoquant une maladie conformationnelle de type «perte de fonction», soit l'accumulation de la protéine cible et, dans ce cas, une pathologie conformationnelle «gain de fonction». La compréhension du processus en cause est cruciale car l'opportunité thérapeutique existe de corriger le défaut de ciblage de la protéine mutante et de proposer un «sauvetage » ou une récupération de fonction, et donc in fine d'anticiper un traitement potentiel de la maladie en question.

\section{Premières observations de chaperons chimiques}

Le premier argument suggérant une possible «manipulation » du système contrôle-qualité du RE vient d'études effectuées sur le canal chlore CFTR (Cystic fibrosis transmembrane conductance regulator) impliqué dans la mucoviscidose. Une baisse de la température du milieu dans lequel les cellules exprimant le mutant CFTR le plus fréquent ( $\Delta \mathrm{F} 508)$ croissent augmente le nombre de canaux fonctionnels à la membrane plasmique. Ce résultat fut interprété comme un effet cinétique, en l'occurrence un ralentissement du procédé de repliement de la protéine, favorisant le sauvetage du mutant $\Delta F 508$. À la suite de cette observation, les effets de petits composés chimiques, tels que le diméthylsulfoxyde (DMSO, solvant organique), le glycérol ou le triéthylamine $\mathrm{N}$-oxyde (TMAO, tous deux définis comme osmolytes cellulaires), connus pour stabiliser la conformation native de plusieurs protéines et s'opposer à la dénaturation thermique ou chimique, furent testés. Les effets sur le mutant $\Delta \mathrm{F} 508$ ont été très probants, ces composés induisant de façon très efficace la maturation de la protéine et son «échappement»au système contrôle-qualité [8]. Le mécanisme d'action de ces molécules n'est pas complètement compris: on pense qu'elles stabilisent une conformation protéique quasi native, et donc favorisent le ciblage des mutants vers le compartiment cellulaire dans lequel ils exercent leur fonction. Le glycérol, par exemple, augmente la stabilité des protéines en diminuant leur surface accessible au milieu aqueux. Ces molécules sont collectivement appelées chaperons chimiques. Aujourd'hui, l'action bénéfique de ces chaperons chimiques a été démontrée in vitro non seulement vis-à-vis du CFTR mais aussi d'autres protéines dont l' $\alpha$ l-antitrypsine, l'ATPase MNK (ATPase à cuivre de Menkes), l' $\alpha$-galactosidase, la p53, le récepteur SURl, l'aquaporine-2, des RCPG. Cependant, dans toutes ces études, des concentrations très élevées (en général micromolaires) de ces chaperons chimiques sont nécessaires pour entraîner un effet, et leur manque de spécificité empêche toute utilisation thérapeutique dans le cadre d'essais cliniques. Par analogie, des peptides cationiques tels que la pénétratine, qui entrent dans les cellules par un phénomène d'endocytose, possèdent la capacité de cibler vers la membrane plasmique des mutants du récepteur V2 de l'hormone anti-diurétique, responsables d'un DINc (diabète insipide néphrogénique congénital) et retenus dans des compartiments post-RE (Ergic ou endoplasmic reticulum-Golgi intermediate compartment par exemple) [9]. Dans cet exemple aussi, des concentrations micromolaires sont nécessaires et la notion de spécificité d'action vis-à-vis de la protéine cible n'est pas présente. Comme pour les chaperons chimiques, le mécanisme d'action précis n'est pas connu. Cependant, ces peptides cationiques entraînent une augmentation cytosolique de calcium et donc modulent l'activité dépendante du calcium de certains chaperons moléculaires du système contrôle-qualité cellulaire [9].

\section{Le concept de chaperon pharmacologique}

L'utilisation de chaperons chimiques a conduit naturellement les scientifiques à rechercher des molécules spécifiques utilisables dans le traitement des maladies conformationnelles chez l'homme. Une première étude fut réalisée à l'aide de mutations artificielles du gène de résistance multidrogues. Celui-ci code pour un transporteur membranaire appelé $\mathrm{P}$-glycoprotéine 1 (MDRl) qui interagit avec une panoplie d'agents cytotoxiques [10]. Les mutants de MDRl sont retenus dans le $R \varepsilon$, mais le traitement des cellules à l'aide de substrats (vinblastine et capsaicine) ou d'inhibiteurs (cyclosporine et vérapamil) du transporteur permet une maturation du MDRl et son ciblage à la membrane plasmique et donc une récupération de fonction. Le mécanisme proposé était le suivant: l'occupation du site actif par des composés spécifiques pourrait stabiliser un état conformationnel quasi natif accepté par le système contrôle-qualité du RE. Ce scénario implique une liaison de composés pharmacologiques (substrats, inhibiteurs) à des mutants mal repliés de protéines pour les stabiliser ; il est similaire à celui des chaperons chimiques mais fait intervenir un paramètre crucial : celui de la spécificité d'action. C'est l'acte de naissance de la notion de chaperons pharmacologiques. Selon ce concept, des ligands qui, en se fixant à des récepteurs connus, stabilisent une conformation spécifique (active, inactive), pourraient être utilisés comme chaperons pharmacologiques vis-à-vis de mutants de RCPG séquestrés dans le RE et responsables de maladies génétiques. Ainsi, l'utilisation d'antagonistes non peptidiques du récepteur V2 de la vasopressine pour neutraliser l'effet de mutations responsables du DINc, par exemple le mutant V2R $\Delta 62-64$ (délétion des résidus aminoacides 62 à 64), fut 


\section{(1) Activité de pharmacochaperon}

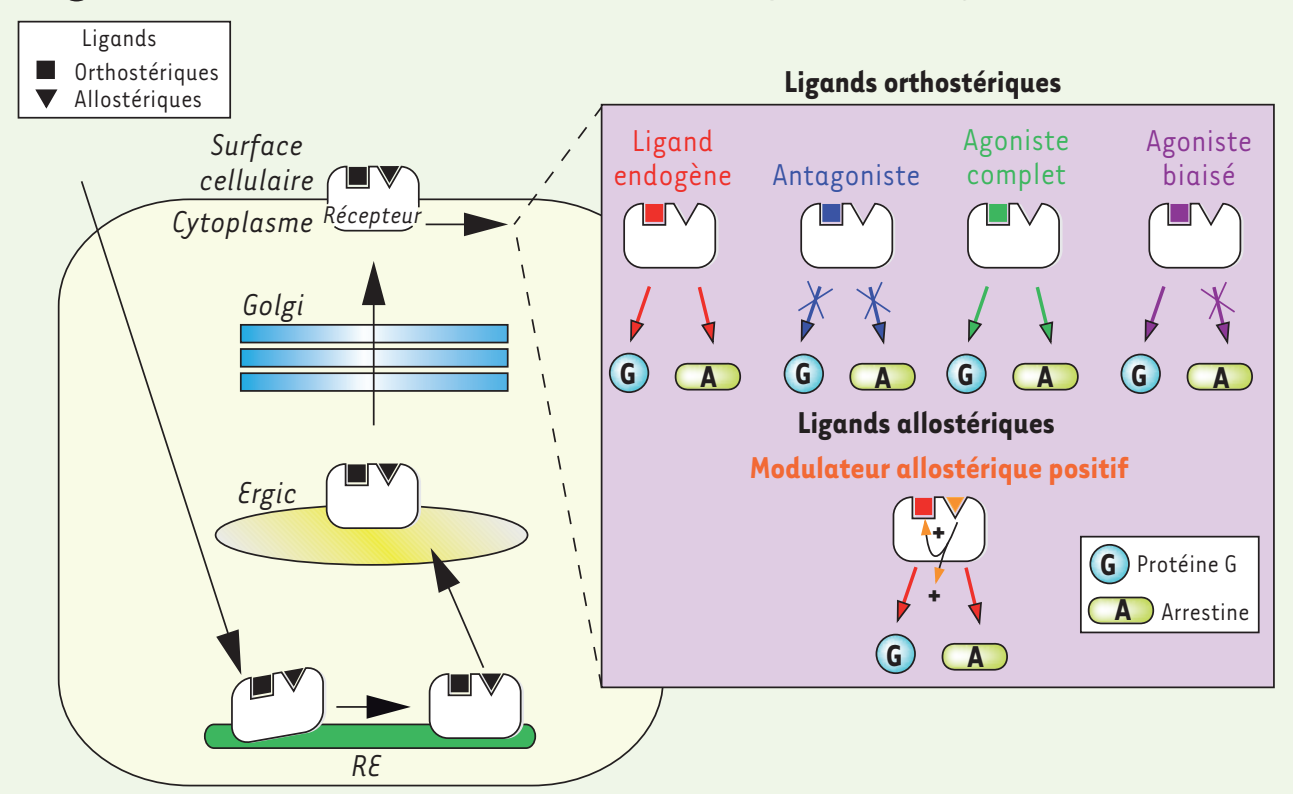

Figure 1. Mode d'action des chaperons pharmacologiques: cas des récepteurs membranaires. Les chaperons pharmacologiques traversent les membranes cellulaires pour interagir avec leurs récepteurs cibles mal repliés et séquestrés au niveau du RE (panneau de gauche). Le changement de conformation induit par le pharmacochaperon permet au récepteur de reprendre son trafic cellulaire classique (à travers l'Ergic et le Golgi) pour subir une maturation complète et être exporté vers son compartiment de fonction (en l'occurrence la membrane

plasmique). Les chaperons pharmacologiques peuvent être de natures différentes. Différentes classes sont décrites: agonistes (activateurs des voies de signalisation, protéines $G$ et arrestines par exemple pour les RCPG), antagonistes (ou agonistes inverses dans certains cas, inhibiteurs des voies de signalisation), agonistes biaisés (activateurs ou inhibiteurs d'une partie des voies de signalisation naturellement engagées par le ligand endogène), modulateurs allostériques positifs (permettent de potentialiser les effets du ligand endogène).

couronnée de succès [11]. Ces antagonistes (ou agonistes inverses, voir définition ci-dessous) - le SR121463, le SR49059, ou le VPA985 par exemple - capables de traverser les membranes cellulaires du fait de leur nature chimique hydrophobe, ont permis une maturation des mutants testés et leur ciblage à la membrane plasmique, entraînant une récupération de fonction - ici leur activation par la vasopressine et leur capacité à se coupler à la protéine Gs et à produire de l'AMPc (adénosine monophosphate, signal intracellulaire). Des antagonistes peptidiques incapables de traverser les membranes cellulaires ne peuvent pas dupliquer ces effets. La stratégie des pharmacochaperons a été appliquée depuis à de nombreuses protéines responsables de plusieurs maladies conformationnelles, mais il s'agit pour l'instant d'études cellulaires in vitro (voir Tableau I/ et Figure 1). Par leur spécificité d'action, les pharmacochaperons exercent leur activité à des concentrations nanomolaires [12-14], ce qui est un avantage supplémentaire par rapport aux chaperons chimiques utilisés à des concentrations cent à mille fois plus élevées. L'utilisation de chaperons pharmacologiques in vivo chez l'homme est très récente. Une étude a été réalisée chez des patients atteints de DINc et a montré que l'administration d'un antagoniste des récepteurs de la vasopressine (SR49059) permettait de diminuer fortement le volume urinaire et la prise d'eau [12]. C'est à ce jour le seul exemple publié d'application thérapeutique de chaperons pharmacologiques chez l'homme, mais il est extrêmement prometteur. La stratégie est reproduite aujourd'hui pour d'autres maladies conformationnelles, en particulier pour le traitement des maladies de Fabry et de Gaucher.

\section{Caractéristiques des chaperons pharmacologiques}

\section{Antagonistes (et agonistes inverses)}

La grande majorité des chaperons pharmacologiques de RCPG décrits aujourd'hui sont des antagonistes (inhibiteurs de récepteurs) et des agonistes inverses (classés tout d'abord comme des antagonistes, ces composés sont capables de diminuer le niveau basal d'activité de leurs récepteurs spécifiques). Dans le cas de l'hypogonadisme hypogonadotrope (Encadré 1), il existe par exemple au moins quatre classes chimiques d'antagonistes pharmacochaperons du récepteur de l'hormone $\mathrm{GnRH}$ (gonadotrophine releasing hormone) (voir Tableau II): indoles, quinolones, macrolides dérivés de l'érythromycine, thiéno[2,3- $\beta$ ] pyrimidine-2,4-diones [15]. Dans le cas de la rétinite pigmentaire, plusieurs antagonistes (agonistes inverses) pharmacochaperons ont été testés : 9-cis-rétinal, 11-cis-rétinal et 11-cis-7-ring rétinal [16]. Aujourd'hui, peu d'agonistes pharmacochaperons (activateurs des récepteurs) sont décrits, même si de nombreuses molécules possèdent intrinsèquement cette propriété [17]. D’un point de vue général, ces différentes catégories de chaperons pharmacologiques possèdent la capacité de traverser les membranes cellulaires pour atteindre leurs protéines cibles bloquées dans le RE ou 


\begin{tabular}{|c|c|c|}
\hline Protéines cibles & Chaperons pharmacologiques spécifiques & Référence \\
\hline \multicolumn{3}{|c|}{ Récepteurs couplés aux protéines G (RCPG) } \\
\hline Rhodopsine & Antagonistes: 9-cis-rétinal, 11-cis-rétinal, 11-cis-7-ring rétinal & {$[24]$} \\
\hline V2 & $\begin{array}{l}\text { Antagonistes : SR121463, SR49059, VPA985, YM087, OPC41061, OPC31260 } \\
\text { Agonistes biaisés: MCF14, MCF18, MCF57 }\end{array}$ & {$[20-23]$} \\
\hline GnRHR & $\begin{array}{l}\text { Antagonistes : indoles, quinolones, macrolides dérivé de l'érythromycine, } \\
\text { thiéno[2,3-b]pyrimidine-2,4-diones }\end{array}$ & [16] \\
\hline Récepteur $\delta$-opioïde $(\delta 0 R) \star$ & $\begin{array}{l}\text { Antagonistes: naltrexone, naltriben, naltrindole, naloxone } \\
\text { Agonistes: buprénorphine, TAN- } 67, \text { SNC-80, brémazocine, tonazocine, nalbuphine }\end{array}$ & [25] \\
\hline $\begin{array}{l}\text { Récepteur } \\
\beta 1 \text {-adrénergique**}\end{array}$ & Antagonistes : alprénolol, carvédilol, labétolol, propranolol & [47] \\
\hline FSHR & Modulateur allostérique : dérivé thiénopyr(im)idine (0rg41841) & [26] \\
\hline \multicolumn{3}{|l|}{ Autres protéines } \\
\hline p53 & Ellipticine, CP-321398, PRIMA-1 & [28] \\
\hline Smo & Cyclopamine, GDC-0449 & {$[48]$} \\
\hline MDRl & Vinblastine, capsaïcine, ciclosporine, vérapamil & [19] \\
\hline Phénylalanine hydroxylase & 3-amino-2-benzyl-7-nitro-4-(2-quinolyl)-1,2-dihydroisoquinoline-1-one & [49] \\
\hline$\beta$-glucosidase acide & $\mathrm{N}$-(n-nonyl)déoxynojirimycine & [12] \\
\hline$\alpha$-galactosidase $\mathrm{A}$ & Galactonojirimycine & {$[50]$} \\
\hline CFTR & VRT-325 (quinazoline), Corr- $2 b$ et Corr- $4 a$ (thiazoles) & {$[51]$} \\
\hline Prion PrP & Quinacrine, chlorpromazine & {$[52]$} \\
\hline Récepteur sulfonylurée SURI & Diazoxide, glibenclamide, tolbutamide & [31] \\
\hline$\beta$-amyloïde & iA $\beta 5$ ( $\beta$-sheet breaker spécifique) & {$[53]$} \\
\hline Huntingtine & Direct fast yellow, chrysamine $\mathrm{G}$ & {$[54]$} \\
\hline$\alpha$-synucléine & $\begin{array}{l}\text { peptide RGGAVVTGRRRRRR-amide } \\
\text { ( } \beta \text {-sheet breaker spécifique) }\end{array}$ & {$[45,46]$} \\
\hline
\end{tabular}

Tableau II. Chaperons pharmacologiques et protéines cibles. *Des mutants naturels du récepteur $\delta$-opioïde n'ont pas été décrits, mais le récepteur est une cible majeure dans le traitement de la douleur. Le $\delta 0 R$ est naturellement mal maturé et $40 \%$ uniquement des récepteurs synthétisés sont ciblés à la surface cellulaire (membrane plasmique). II y a donc un intérêt thérapeutique réel à développer des chaperons pharmacologiques de ce récepteur [25]. **Le récepteur $\beta 1$-adrénergique n'est pas impliqué dans des maladies conformationnelles, mais les chaperons pharmacologiques spécifiques sont capables de cibler des mutants artificiels de ce récepteur à la surface cellulaire [47].

dans un autre compartiment intracellulaire (Figure 1). Ces molécules sont donc plutôt de nature lipophile, et leur activité de pharmacochaperon ne peut pas être reproduite par des ligands non perméants. La restauration de la fonction d'une protéine mutante dépend bien sûr de la mutation elle-même et de ses conséquences sur la conformation globale. Mais de façon intéressante, il existe une corrélation entre l'affinité (force d'interaction) du chaperon pharmacologique pour le récepteur et la récupération de la fonction protéique. Plus l'affinité est forte, plus l'effet est important. Intuitivement, cette corrélation ne va pas de soi pour les antagonistes et les agonistes inverses. En effet, que le chaperon agisse comme antagoniste ou agoniste inverse, la récupération de la fonction protéique est la résultante d'un équilibre subtil entre la force du caractère chaperon et la possibilité qu'il soit déplacé par le ligand endogène naturel activateur. C'est tout le paradoxe de ces ligands qui permettent un ciblage des récepteurs mutants à la surface cellulaire mais qui sont dans l'impossibilité de stimuler 
directement la protéine dont ils sont inhibiteurs. Pour une application thérapeutique, il semblerait donc plus approprié de sélectionner des chaperons pharmacologiques antagonistes et agonistes inverses possédant une affinité modérée pour leur cible. Un exemple qui illustre bien ce concept est celui de l'antagoniste SR49059 non spécifique du récepteur V2 (mais spécifique du récepteur Vla de la vasopressine) et pour lequel il a une affinité très moyenne. Cependant, ce ligand est capable de cibler des mutants du V2R à la surface cellulaire et d'induire un effet antidiurétique chez des malades atteints de DINc [12].

\section{Agonistes}

Logiquement, les chaperons pharmacologiques agonistes seraient donc plus intéressants puisque capables à la fois de cibler les récepteurs mal repliés à la surface et de les stimuler directement (Figure 1). Dans ce cas, une forte affinité est un avantage, et ces molécules peuvent remplacer avantageusement le ligand naturel activateur. Mais les agonistes présentent aussi des inconvénients. Leur liaison avec le récepteur cible provoque son activation mais également son internalisation via une interaction avec une protéine régulatrice, l'arrestine. L'internalisation, qui fait intervenir un mécanisme d'endocytose, régule le nombre de récepteurs à la surface cellulaire et donc l'intensité du signal intracellulaire. Une fois le récepteur internalisé, il n'est plus accessible au ligand activateur. Ce phénomène (appelé aussi processus de désensibilisation) conduit à l'arrêt progressif de la réponse cellulaire. Des agonistes du récepteur $\delta$-opioïde $(\delta 0 R)$ et d'un mutant artificiel de cette protéine (D95A), possédant une activité de pharmacochaperon, ont été décrits mais ils ont également la capacité d'induire l'internalisation du SOR via l'arrestine [17].

\section{Agonistes biaisés}

La découverte très récente d'agonistes biaisés du V2R (MCF14, MCF18, MCF57) offre une nouvelle opportunité thérapeutique dans le DINC [13]. Un agoniste biaisé est une molécule qui ne possède qu'une partie des propriétés pharmacologiques du ligand activateur endogène. En l'occurrence, les chaperons pharmacologiques MCF14, MCF18 et MCF57 de plusieurs mutants du V2R sont des activateurs du récepteur: ils provoquent l'activation de la protéine Gs, mais n'entraînent pas son internalisation parce qu'ils s'opposent au recrutement de l'arrestine (Figure 1). Ils offrent donc un avantage certain par rapport aux agonistes classiques et aux antagonistes, tout en ayant une affinité forte vis-à-vis du V2R. L'association de ces caractéristiques semble idéale pour traiter les patients atteints de DINc puisque ces molécules induisent effectivement le ciblage de mutants du V2R à la surface cellulaire [13]. Ces composés devraient permettre a priori une activation soutenue et durable dans le temps des V2R mutés puisque ceux-ci ne sont pas internalisés. On peut imaginer, même si cela reste à démontrer, un traitement par voie orale peu contraignant pour les malades, et espérer une régulation efficace de la diurèse.

\section{Modulateurs allostériques}

Enfin, il nous faut citer une dernière catégorie de molécules, les modulateurs allostériques, qui pourraient également être des cha- perons pharmacologiques tout aussi prometteurs. Un modulateur allostérique se fixe sur sa protéine cible de façon spécifique, mais dans un site d'interaction différent de celui du substrat naturel (pour les enzymes) ou du ligand endogène (pour les récepteurs ou les canaux ioniques). Sa liaison à la protéine entraîne un changement de conformation qui peut avoir une influence positive ou négative sur la liaison de l'activateur (ligand, substrat), sur la capacité de la protéine à produire un effet biologique, ou sur les deux (Figure 1). II n'y a qu'un exemple aujourd'hui de modulateur allostérique pouvant agir comme chaperon pharmacologique. Cette molécule est spécifique du récepteur de l'hormone FSH (follicle stimulating hormone) [18]. Lorsqu'elle se fixe sur son récepteur, elle participe à la régulation de la maturation des follicules ovariens chez la femme et contrôle la spermatogenèse chez I'homme. Plusieurs mutations du récepteur de la FSH ont été découvertes qui provoquent des troubles endocriniens. La mutation Al89V bloque le récepteur dans le $R \varepsilon$ et empêche son ciblage à la membrane plasmique. Un composé activateur spécifique du récepteur a été découvert, mais il n'est pas un ligand compétiteur de l'hormone naturelle. Ce composé (Org41841, famille des thiénopyr(im)idines) permet l'acheminement du récepteur mutant à la surface cellulaire où il peut être activé et induire une réponse de la cellule. Dans ce cas, Org4184l et FSH agissent de concert: Org4184l favorise l'activité de l'hormone FSH. Ce type de molécule possède donc des caractéristiques tout à fait particulières, c'est un modulateur allostérique positif avec une activité de chaperon pharmacologique.

\section{Perspectives}

Le concept de chaperon pharmacologique découle directement de l'idée que toute mutation dans une protéine n'entraîne pas obligatoirement une perte de fonction, à condition que le défaut mineur puisse être sélectivement compensé. En effet, beaucoup de protéines mutées pourraient être actives mais sont tout simplement rejetées par le système contrôle-qualité du RE et ne sont pas exportées dans leur compartiment cellulaire final. Or, un nombre croissant d'exemples démontrent que les chaperons pharmacologiques facilitent le repliement et la maturation de leurs protéines cibles, in vitro comme in vivo, y compris chez des patients. II est donc probable que cette stratégie thérapeutique puisse être applicable à une majorité de maladies conformationnelles. Ces outils thérapeutiques prometteurs concernent des pathologies conformationnelles aussi bien «perte de fonction» que «gain de 
fonction » en prévenant l'agrégation des protéines mal repliées. De plus, le fait qu'un chaperon pharmacologique permette une récupération de fonction de plusieurs mutants d'une même protéine spécifique devrait simplifier le développement de médicaments ou drogues utiles pour traiter les maladies conformationnelles. En d'autres termes, il n'est pas nécessaire de développer un médicament chaperon pharmacologique pour chaque mutation (chaque patient). Ceci est un élément important qui devrait inciter les industriels à s'investir plus activement dans la mise au point de ces molécules.

De façon intéressante, les chaperons pharmacologiques agissent sur des protéines dont beaucoup sont des cibles thérapeutiques majeures (récepteurs, canaux, transporteurs, enzymes). De multiples ligands avec une forte affinité et sélectivité sont déjà identifiés ou en développement. Ces ligands sont des pharmacochaperons en devenir, à la condition qu'ils soient capables de traverser les membranes cellulaires pour atteindre leurs cibles bloquées dans le RE ou dans d'autres compartiments (Ergic, Golgi). Les futures campagnes de criblage de chaperons pharmacologiques devront donc tirer parti de chimiothèques déjà disponibles et dont les cibles sont bien définies. La recherche d'agonistes biaisés et de modulateurs allostériques positifs sera particulièrement attractive. Le fait que de très nombreuses molécules soient déjà en développement, même si leur caractère pharmacochaperon n'est pas pour l'instant la propriété thérapeutique mise en avant, est vraiment un avantage. Ces médicaments à caractère pharmacochaperon potentiel seront développés en priorité pour des pathologies majeures bien définies. Mais ils pourraient aussi être testés en parallèle sur des patients volontaires atteints de maladies orphelines, lorsque leur processus de développement clinique sera suffisamment avancé (phase III), et ce malgré la réticence des entreprises pharmaceutiques à mettre sur le marché des médicaments pour les maladies orphelines. $\diamond$

\section{SUMMARY}

Pharmacological chaperones:

a potential therapeutic treatment

for conformational diseases

Many genetic and neurodegenerative diseases in humans result from protein misfolding and/or aggregation. These diseases are named conformational diseases. As a result, the misfolded non functional proteins are rejected and misrouted by the cellular quality control system, and cannot play their endogenous physiological roles. Specific compounds (ligands, substrates or inhibitors) known as pharmacological chaperones are able to bind and stabilize these misfolded proteins. Their interaction allows the target proteins to escape the quality control system and to be functionally rescued. These pharmacochaperones may possess different intrinsic activity: they can be antagonists (inhibitors), agonists (activators) or allosteric modulators of the target receptors, ionic channels or enzymes. Pharmacological chaperones have obviously a therapeutic potential to treat rare diseases like cystic fibrosis, retinitis pigmentosa, nephrogenic diabetes insipidus, Fabry disease, Gaucher disease, but also for cancers and more frequent and highly invalidant neurodegenerative disorders such as Alzheimer's disease or Parkinson's disease. $\diamond$

\section{REMERCIEMENTS}

Les auteurs remercient Muriel Asari-Gien pour la réalisation iconographique de cet article.

\section{CONFLIT D'INTÉRÊTS}

Les auteurs déclarent n'avoir aucun conflit d'intérêts concernant les données publiées dans cet article.

\section{RÉFÉRENCES}

1. Ellgaard L, Helenius A. Quality control in the endoplasmic reticulum. Nature Rev Mol Cell Biol $2003 ; 4: 181-91$.

2. Anelli T, Sitia R. Protein quality control in the early secretory pathway. EMBO J $2008 ; 27: 315-27$.

3. Arrigo AP. Chaperons moléculaires et repliement des protéines. Med Sci (Paris) $2005 ; 21: 619-25$.

4. Tsai $B$, Ye Y, Rapoport TA. Retro-translocation of proteins from the endoplasmic reticulum into the cytosol. Nature Rev Mol Cell Biol 2002 ; 3 : 246-55.

5. Cohen FE, Kelly JW. Therapeutic approaches to protein-misfolfding diseases. Nature 2003; 426: $905-9$.

6. Chaudhuri TK, Paul S. Protein-misfolding diseases and chaperone-based therapeutic approaches. FEBS J 2006 ; 273 : 1331-49.

7. Conn PM, Ulloa-Aguirre A, Ito J, Janovick JA. G protein-coupled receptors trafficking in health and disease: lessons learned to prepare for therapeutic mutant rescue in vivo. Pharmacol Rev 2007 ; $59: 225-50$.

8. Sato S, Ward CL, Krouse ME, et al. Glycerol reverses the misfolding phenotype of the most common cystic fibrosis mutation. J Biol Chem 1996; $271: 635-8$.

9. Oueslati $M$, Hermosilla R, Schönenberger $\varepsilon$, et al. Rescue of a nephrogenic diabetes insipiduscausing vasopressin V2 receptor mutant by cell-penetrating peptides. J Biol Chem 2007; $282: 20676-85$.

10. Loo TW, Clarke DM. Correction of defective protein kinesis of human P-glycoprotein mutants by substrates and modulators. J Biol Chem $1997 ; 272$ : 709-12.

11. Morello JP, Salahpour A, Laperrière A, et al. Pharmacological chaperones rescue cell-surface expression and function of misfolded V2 vasopressin receptor mutants. J Clin Invest 2000; $105: 887-95$.

12. Bernier V, Morello JP, Zarruk A, et al. Pharmacologic chaperones as a potential treatment for Xlinked nephrogenic diabetes insipidus. J Am Soc Nephrol 2006 ; 17 : 232-43.

13. Jean-Alphonse F, Perkovska S, Frantz MC, et al. Biased agonist pharmacochaperones of the AVP V2 receptor may treat congenital nephrogenic diabetes insipidus. J Am Soc Nephrol 2009; $20: 2190-203$.

14. Robben JH, Sze M, Knoers NV, Deen PM. Functional rescue of vasopressin V2 receptor mutants in MDCK cells by pharmacochaperones: relevance to therapy of nephrogenic diabetes insipidus. Am J Physiol Renal Physiol 2007 ; 292 : F253-60.

15. Conn PM, Janovick JA. Drug development and the cellular quality control system. Trends Pharmacol Sci $2009 ; 30: 228-33$.

16. Li T, Sandberg MA, Pawlyk BS, et al. Effect of vitamine A supplementation on rhodopsin mutants T17M and P347S in transgenic mice and in cell cultures. Proc Natl Acad Sci USA 1998 ; 95 : 11933-8.

17. Petäjä-Repo UE, Hogue M, Bhalla S, et al. Ligands act as pharmacological chaperones and increase the efficiency of $\delta$ opioid receptor maturation. EMBO J $2002 ; 21: 1628-37$.

18. Janovick JA, Maya-Nunez G, Ulloa-Aguirre A, et al. Increased plasma membrane expression of human follicle-stimulating hormone receptor by a small molecule thienopyr(im)idine. Mol Cell Endocrinol $2009 ; 298: 84-8$.

19. Riordan JR. The cystic fibrosis transmembrane conductance regulator. Annu Rev Physiol 1993; $55: 609-30$. 
20. Kerem B, Rommens JM, Buchanan JA, et al. Identification of the cystic fibrosis gene: genetic analysis. Science $1989 ; 245$ : 1073-80.

21. Germain DP. La maladie de Fabry : de la découverte des lysosomes à l'avènement de la thérapeutique. Med Sci (Paris) $2005 ; 21: 5-7$.

22. Fan JQ, Ishii S, Asano N, Suzuki Y. Accelerated transport and maturation of lysosomal alphagalactosidase A in Fabry lymphoblasts by an enzyme inhibitor. Nat Med $1999 ; 5: 112-5$.

23. Grabowski GA. Gaucher disease: enzymology, genetics and treatment. Adv Hum Genet 1993; $21: 377-441$.

24. Sawkar AR, Cheng WC, Beutler $\varepsilon$, et al. Chemical chaperones increase the cellular activity of N370S beta-glucosidase: a therapeutic strategy for Gaucher disease. Proc Natl Acad Sci USA 2002 ; 99: 15428-33.

25. Mendes HF, Van der Spuy J, Chapple JP, Cheetham ME. Mechanisms of cell death in rhodopsin retinitis pigmentosa : implications for therapy. Trends Mol Med $2005 ; 11: 177-85$.

26. Morello JP, Bichet DG. Nephrogenic diabetes insipidus. Annu Rev Physiol 2001 ; 63 : 607-30.

27. Carrell RW, Lomas DA, Sidhar S, Foreman R. alphal-antitrypsin deficiency: a conformational disease. Chest $1996 ; 110: 2435-475$.

28. Foster BA, Coffey HA, Morin MJ, Rastinejad F. Pharmacological rescue of mutant p 53 conformation and function. Science $1999 ; 286: 2507-10$.

29. Toledo F, Bluteau 0 , Simeonova I. Réactivation de $p 53$ dans les tumeurs : une stratégie antitumorale prometteuse. Med Sci (Paris) 2007 ; $23: 565-7$.

30. Lam CW, Xie J, To KF, et al. A frequent activated smoothened mutation in sporadic basal cell carcinomas. Oncogene $1999 ; 18: 833-6$.

31. Partridge CJ, Beech DJ, Sivaprasadarao A. Identification and pharmacological correction of a membrane trafficking defect associated with a mutation in the sulfonylurea receptor causing familial hyperinsulinism. J Biol Chem $2001 ; 276: 35947-52$.

32. Pratt EB, Yan FF, Gay JW, et al. Sulfonylurea receptor 1 mutations that cause opposite insulin secretion defects with chemical chaperone exposure. J Biol Chem 2009 ; 284 : 7951 -9.

33. Kim BE, Smith K, Meagher CK, Petrisd MJ. A conditional mutation affecting localization of the Menkes disease copper ATPase; suppression by copper supplementation. J Biol Chem 2002 ; $277: 44079-84$

34. Liu X, Garriga P, Khorana HG. Structure and function in rhodopsin : correct folding and misfolding in two point mutants in the intradiscal domain of rhodopsin identified in retinitis pigmentosa. Proc Natl Acad Sci USA $1996 ; 93:$ : 4554-9.

35. Fuchs S, Amiel J, Claudel S, et al. Functional characterization of three mutations of the endothelin $B$ receptor gene in patients with Hirshsprung's disease: evidence for selective loss of Gi coupling. Mol Med $2001 ; 7: 115-24$.

36. Lubrano-Berthelier C, Dubern B, Lacorte JM, et al. Melanocortin 4 receptor mutations in a large cohort of severely obese adults: prevalence, functional classification, genotype-phenotype relationship, and lack of association with binge eating. J Clin Endocrinol Metab 2006; 91 : 1811-8.

37. Clark AJL, Metherell LA, Cheetham ME, Huebner A. Inherited ACTH insensitivity illuminates the mechanisms of ACTH action. Trends Endocrinol Metab 2005; 16:451-7.

38. Samson M, Libert F, Doranz BJ, et al. Resistance to HIV-1 infection in caucasian individuals bearing mutant alleles of the CCR-5 chemokine receptor gene. Nature $1996 ; 382: 722-5$.

39. Tfelt-Hansen J, Brown EM. The clacium-sensing receptor in normal physiology and athophysiology: a review. Crit Rev Clin Lab Sci $2007 ; 42: 35-70$.
40. Biebermann H, Schoneberg T, Krude H, et al. Mutations of the human thyrotropin receptor gene causing thyroid hypoplasia and persistent congenital hypothyroidism. J Clin Endocrinol Metab 1997 ; 82 : 3471-80.

41. Themmen APN, Martens JWM, Brunner HG. Activating and inactivating mutations in LH receptors. Mol Cell Endocrinol 1998; 145 : 137-42.

42. Aittomäki KJ, Lucena LD, Pakarinen P, et al. Mutation in the folliclestimulating hormone receptor gene causes hereditary hypergonadotropic ovarian failure. Cell 1995; 82 : 959-68.

43. Beaumont KA, Newton RA, Smit DJ, et al. Altered cell surface expression of human MCRl variant receptor alleles associated with red hair and skin cancer risk. Hum Mol Genet $2005 ; 14: 2145-54$.

44. Noorwez SM, Malhotra R, McDowell JH, et al. Retinoids assist the cellular folding of the autosomal dominant retinitis pigmentosa opsin mutant $\mathrm{P} 23 \mathrm{H}$. J Biol Chem 2004; 279 : 16278-84.

45. Lees AJ, Hardy J, Revesz T. Parkinson's disease. Lancet 2009 ; 373 : 2055-66.

46. Sacchetini JC, Kelly JW. Therapeutic strategies for human amyloid diseases. Nat Rev Drug Discov $2002 ; 1: 267-75$.

47. Kobayashi H, Ogawa K, Yao R, et al. Functional rescue of beta-adrenocepto dimerization and trafficking by pharmacological chaperones. Traffic 2009; $10: 1019-33$.

48. Stanton BZ, Peng LF. Small molecule modulators of the Sonic Hedgehog signalling pathway. Mol Biosyst $2010 ; 6: 44-54$.

49. Pey AL, Ying M, Cremades N, et al. Identification of pharmacological chaperones as potential therapeutic agents to treat phenylketonuria. J Clin Invest 2008 ; 118 : 2858-67.

50. Asano N, Ishii S, Kizu H, et al. In vitro inhibition and intracellular enhancement of lysosomal alpha-galactosidase A activity in Fabry lymphoblasts by 1-deoxygalactonojirimycin an dits derivatives. Eur J Biochem $2000 ; 267: 4179-86$.

51. Loo TW, Bartlett MC, Wang Y, Clarke DM. Rescue of deltaF508 and other misprocessed CFTR mutants by a novel quinazoline compound. Mol Pharmacol $2005 ; 2: 407-13$

52. Korth C, May BC, Cohen FE, Prusiner SB. Acridine and phenothiazine derivatives as pharmacotherapeutics for prion disease. Proc Natl Acad Sci USA 2001 ; 98 : 9836-41.

53. Sigurdsson EM, Permanne $B$, Soto $C$, et al. In vivo reversal of amyloid-beta lesions in rat brain. J Neuropathol Exp Neurol $2000 ; 59: 11-7$.

54. Heiser V, Scherzinger $\varepsilon$, Boeddrich A, et al. Inhibition of huntingtin fibrillogenesis by specific antibodies and small molecules: implications for huntington's disease therapy. Proc Natl Acad Sci USA 2000 ; 97 : 6739-44.

\section{TIRÉS À PART}

C. Mendre et B. Mouillac 